\title{
Considering Every Available Instance: The Early Development of a Fundamental Problem Solving Skill
}

\author{
Henry M. Wellman \\ University of Michigan, U.S.A. \\ William V. Fabricius \\ University of Georgia, U.S.A. \\ Wan Chuan-Wen \\ Chinese Academy of Science, China
}

\begin{abstract}
Two studies are reported describing the early development in two- and three-year-old children of an ability to consider every one of an array of instances. Children were tested on several tasks unlike either counting or searching tasks. Young children, by about three years of age, attempted to consider each item once and only once. They did so by employing a strategy of sequencing the instances so as to consider each in turn. Employment of this strategy became increasingly skilful over the ages studied, so that with increasing age larger problem sizes and more difficult problems were accomplished successfully. Taken together with recent studies of children's ability to count every item or search all locations for a hidden object, the data reveal early development in preschool children of a fundamental, general problemsolving skill.
\end{abstract}

\section{INTRODUCTION}

Problem-solving endeavours have a means-end structure where final performance depends on intermediate steps. A step in much problem

\footnotetext{
Requests for reprints should be sent to H. M. Wellman, Center for Human Growth and Development, 300 North Ingalls Building, The University of Michigan, Ann Arbor, MI 48109.

This research was supported by NICHD grant HD-13317 to the first author, and a visiting scholar fellowship from the Foundation for Child Development to the third author. We wish to thank Gail Kopin and Galley Kronenberg for their help in collecting the data, and the parents, children and staff of the University of Michigan's Children Center for their cooperation and participation.
}

(C) 1987 The International Society for the Study of Behavioral Development 
solving is the systematic consideration of multiple alternatives, terms, or instances, which is evident in activities as diverse as counting, selecting chess moves, or the experimental design of research. In such cases success and efficiency require that one include, check, or transform alternatives once and only once. The simplest manifestation of such a skill involves a set of perceptually present items where each must be checked once; it is required, for example, in counting (e.g. counting every person), searching (searching each room in the house), sorting (putting each sock with its mate), and simple forms of keeping track (checking each item off a list). This activity can be termed considering every available instance, and can be thought of as representing the lower end of a logical continuum of similar skills. An intermediate level of the continuum might include considering every imagined instance, as in using a shopping list. In this case the items are not perceptually available at once, but must be first imagined and then considered. The upper end of the continuum would involve consideration of every imaginable instance. Tasks involving consideration of all possible combinations provide an example of this sort (Inhelder \& Piaget, 1958). In these cases a determinate set of possibilities must be imagined, based on all of the specified variables, and then each of these instances (i.e. combinations) must be tested. The focus of this paper is young children's acquisition of skill at considering every available instance. We suspect that for young children considering every available instance is a general problem-solving skill (utilisable in counting, searching, sorting, etc.) that is instrumental to the acquisition of more advanced skills in considering multiple alternatives.

What evidence is there concerning the acquisition of an ability to consider every available instance in young children? Gelman and Gallistel (1978) showed that young preschoolers can often consider every instance in counting (their one-one principle). In that research, three-year-olds apparently realised that each object should be counted once and only once, although they were typically unable to do so perfectly if more than three or four items were involved. Skill at executing this goal with larger, more complex arrays developed rapidly in the preschool years. Similarly, Wellman et al. (1984) found that three-year-olds would attempt to search each potential hiding location once and only once to find an object, and indeed often performed essentially at ceiling with eight locations. It thus appears that some competence at considering every available instance is evident quite early in development. However, Cornell and Heth (1983) suggest that search performances of this sort are due to specially evolved spatial capacities, since abilities to search for food would have clear survival value. Similarly, Starkey, Spelke and Gelman (1980) propose that numerical abilities may be a "natural domain of competence". One issue, then, is whether young children are simply learning to count and to search or whether they have acquired a more general skill at considering every 
instance. If they have, then such a skill should be evident in other tasks, ones whose goals are not to achieve an enumeration nor to find a missing object.

A second issue concerns whether young children's counting and searching reflect a deliberate ability to consider every instance, or reflect instead non-deliberate response tendencies. One previous study sheds light on both these issues (Potter \& Levy, 1968). Two-, three-, and four-year-olds were asked to simply touch every object drawn on a page and were able to do so under some conditions. Potter and Levy concluded that children used one of two strategies: (1) making unguided choices of an instance coupled with a memory check to see if the instance had been touched before; (b) following a spatial plan, i.e. ordering the instances based on their spatial configuration and then proceeding to each instance in sequence. The youngest subjects seemed to use the memory-only strategy, while somewhat older children used the sequencing approach. Evidence of a sequencing strategy was that children tended to touch each item in a spatial order. But: "the only spatial principles used by most children were to start touching the corner item nearest to the child's hand and to proceed by touching adjacent items" (Potter \& Levy, 1968, p. 271). This pattern of response may represent only an adjacent neighbour response tendency, based on moving the hand to whatever is next closest. The evidence for a memory-only strategy was that younger children tended to do better on a random array of objects than on a rectangular array-items in a random array, while less orderly, are more spatially distinct and hence more memorable. But the cited advantage occurred only when the array contained six items; so few items can easily result in an orderly spatial configuration themselves.

This discussion suggests that determining the nature of children's efforts requires distinguishing three different aspects of performance. One must distinguish (1) whether children are attempting to consider every instance, from (2) their success at doing so. Intending to do something does not ensure success and success does not necessarily require intention. One must also distinguish both success and goals from (3) the child's strategies-the procedures that are recruited in order to achieve the goal with some level of success. For considering every available instance, reasonable strategies require some procedure aimed at keeping track of items so that none is skipped and none done twice. Specific strategies, however, can differ in success.

In the present research we examine young children's competence at considering every available instance in tasks which do not require them to achieve a count nor to find an object. This will inform us about the generality versus embeddedness of a skill which, if acquired early and generally, may prove a specially useful problem solving competence.

BD $10: 4-\mathrm{G}$ 


\section{EXPERIMENT 1}

Children performed two tasks, one where they were to take an object out of each of several containers, and the other where they were to put an object into each container. To what extent are these tasks unlike counting and searching? Both seem different from counting since the goals are so different; the child is never asked to, or needs to, enumerate the items. Similarly, the child's activities are quite different from counting; she or he must execute concrete actions with each instance (emptying or filling), but does not tag the instances with symbolic terms $(1,2,3 \ldots)$ nor tag them with other sorts of markers. Both tasks require that the child consider each instance once only and thus involve one-to-one correspondence, like counting. This is necessary by definition; any possible form of considering instances once and only once requires some one-to-one correspondence of acts and instances. Except for this general and necessary similarity, the other distinctive features of counting tasks were eliminated in the put-in and take-out tasks used here.

The tasks are also unlike searching; the goal was never to find missing objects. The take-out task, however, appears somewhat like a search task since there are containers concealing objects and objects to be retrieved. However, the child knows there is an object in every container (the task is not to find those containers with objects) and the containers themselves are obvious and visible. Thus, rather than not knowing where the objects are and having to search for them, the child knows exactly where they are and simply must collect them.

The two tasks were designed so that success could result from an adjacent-neighbour response tendency on one task but not the other. In the put-in task, children took pennies one at a time from a bowl and put them into each of several containers. The bowl of pennies was distant from the array of containers so that after putting a penny in container $n$ the child had to take his hand away from the array to get the penny for $n+1$. Removing the hand from the array at this point should disrupt an adjacent neighbour response tendency, since the "next" location in any spatial sequence was no longer adjacent to the hand. In the take-out task, when the child had to proceed from location $n$ to $n+1$ his hand rested on location $n$. Thus, proceeding adjacently was unobstructed.

Like Potter and Levy, problems differed in the number (6 and 12) of instances and also in the arrays (line, circle, and random). Linear arrays include natural starting and stopping points (the ends) and a clear sequence; circular arrays include a sequence without endpoints; truly random arrays include neither sequence nor endpoints. A sequencing strategy should be differentially effective across these arrays (Beckwith \& Restle, 1966), with a linear array clearly easiest to consider. Finally, on one 
take-out and one put-in trial the containers used for the task were transparent; the child could keep track of which containers had and had not been considered simply by looking. These problems served as a check on children's ability to understand the instructions and to solve the task under minimally demanding circumstances.

\section{Method}

Subjects. There were 12 two-year-olds (aged 2.5-2.11 years, $M=2.8$ years) and 12 three-year-olds $(3.0-3.6, M=3.3)$, who attended a racially mixed but predominantly white middle class preschool.

Tasks. For the put-in tasks, small (5cm tall) plastic film containers with a slot in each lid were used. The child's task was to "put a penny (a washer) in every bank". The containers were glued to a board to be stationary. The child received one penny at a time from the adult who held a bowl of pennies away from the array of containers. For the take-out tasks, unlidded film containers were used, each holding one small black "pea" (a piece of soft black sponge). The task was to "empty the pea from every jar". Containers fit into shallow $(0.5 \mathrm{~cm})$ wells, where each was always returned upon being emptied. Thus at the point where the child must decide where to go next, his or her hand was in the spatial array and on the jar just emptied. The containers were black and opaque so that whether they possessed a pea or a penny was difficult to determine by looking. ${ }^{1}$

In the linear and circular arrays, containers were spaced at $6 \mathrm{~cm}$ intervals (from container centre to centre). Random arrays were constructed by randomly sampling 6 or 12 cells from a $5 \times 9$ cell grid. The midpoint of each cell was $6 \mathrm{~cm}$ from the midpoint of the adjacent cells.

These factors form a 2 (age) $\times 2$ (task: put-in, take-out) $\times 3$ (array: line,

\footnotetext{
${ }^{1}$ Using a black pea in a black container for the take-out task hindered looking but, as the data revealed, did not eliminate it completely. Children attempted to look into the "banks" of the put-in task less that $1 \%$ of the time; however, they attempted to look into the "jars" of the take-out task approximately $40 \%$ of the time. These looks were easy to detect since they required the child to put his or her eye directly over the opening of the small container. Since the child might potentially garner some information from looking, in both tasks all looks were counted as actual attempts to execute the relevant action. Proportion-correct calculated in this fashion is a conservative measure of children's performance (since actually seeing the pea was improbable). To the extent that looking was attempted more often in the take-out task, correct responding could be underestimated there relative to the put-in task. This potential bias proved unimportant however, since: (1) take-out performance exceeded put-in performance (rather than the reverse); (2) in Experiment 2 all containers were extremely and equally difficult to look into, and looks were infrequent (less than $5 \%$ of all acts), yet comparable results were obtained.
} 
circle, random) $\times 2$ (number: 6,12 ) design. All factors except age were within subjects. In addition to these tasks with opaque containers, each child received a transparent version of each task. Here the containers were of clear plastic and the presence or absence of peas or pennies was immediately obvious. The intent was to present a minimally demanding version of the task. So that the demands of the problem would indeed be minimal, transparent problems used only six containers. These were arranged in a random configuration. Children were tested in two sessions, one for the put-in and one for the take-out tasks, generally administered on separate days. Task order was counterbalanced across children.

Procedures. The nature of the task was first demonstrated with three transparent containers, then with three opaque containers and a Mickey Mouse doll holding a separate container. The child was told to put a penny in every bank (empty a pea from every jar) and then to put a penny in Mickey's. Putting a penny in (or emptying a pea from) Mickey's terminated each child's performance, providing a simple way to signal completion.

The child and adult sat facing the array, with Mickey on the opposite side of the child from the adult. Left-right position of Mickey (and adult) was counterbalanced across children within an age group. Children received pennies from the adult one at a time and emptied peas into a bowl held by the adult. In each session one transparent and six opaque (3 array $\times 2$ number) problems were administered. The order of these trials was randomised for each child with the constraint that two identical array types (line, circle, random) did not appear in succession. A second adult standing behind the child recorded the sequence of responses. Whether the child put in or took out an item was recorded, as well as whether he or she touched the container without putting anything in or taking anything out. Finally, if the child attempted to look into an opaque container, by peering directly into the opening, that also was recorded.

\section{Results}

Distinguishing children's goals from their strategies and success is important, but empirically doing so can be difficult. Without some demonstration of a strategy for doing $\mathrm{X}$, or of successful accomplishment of $\mathrm{X}$, there is no evidence that a child was nonetheless attempting to do $X$. Thus, transparent problems were included as a check on whether children were attempting to consider every instance or not. Our assumption was that given minimally demanding problems-where visual inspection was sufficient to keep track of considered and to-be-considered instances-any attempt to consider each one should result in reasonable success. Most importantly, if children were attempting to consider every instance then success should be 
equally evident on both the take-out and put-in problems; success on take-out problems alone might result from an adjacent neighbour response tendency. Eleven of twelve two-year-olds successfully considered every instance (each container emptied once and only once) on the transparent take-out problem; only one of twelve was similarly successful on the transparent put-in problem. Ten of twelve three-year-olds were successful on the transparent take-out problem; ten of twelve were successful on the transparent put-in problem as well.

This suggests that three-year-olds were attempting to consider every instance once, though two-year-olds were not. Corroboration for this conclusion comes from analysing children's self-corrections on the opaque problems. At times children would begin executing the relevant act at an opaque container, but then proceed to another container without completing the act at the first. In the put-in task they would touch a container with the penny, not place it in that container, but move on to and place the penny in a different container. In the take-out task they would touch a container as if to pick it up, but then pick up and empty another instead. Self-corrections of this sort occurred infrequently ( $7 \%$ of the time), but they typically resulted in the child avoiding a potentially redundant response. Note that when such self-corrections occurred they did not provide the child any new information (e.g. the child did not actually attempt to pour out the pea and find it missing before moving on). Therefore, if such acts in fact avoid redundancies they evidence some recognition and deliberate avoidance of instances already considered. Self-corrections are therefore important, even though infrequent, as an index of whether children attempt to consider instances only once. The proportion of self-corrections which avoided redundancy differed between the two ages- $\mathrm{F}(1,21)=6.13, P<0.025$. Two-year-olds' self-corrections avoided redundancy $68 \%$ of the time (not significantly different from chance); three-year-olds' did so $94 \%$ of the time (different from chance at the 0.001 level).

Given that children attempt to consider every instance, we can ask how successful they were. Performance on opaque problems provides this data. Note that completely successful performance was not expected on opaque problems, since the design systematically manipulated factors intended to influence both task difficulty and possible strategies. To parametrically analyse all factors in the design, proportion correct scores were used: the proportion of the total instances considered once and only once. ${ }^{2}$ The data for both ages are presented in Table 1 .

A 2 (age) $\times 2$ (task) $\times 3$ (array) $\times 2$ (number) ANOVA on proportion correct yielded effects of number $F(1,22)=40.23$, Mean Square Error

\footnotetext{
${ }^{2}$ See Footnote 1.
} 
TABLE 1

Proportion Correct and Adjacency in Experiment 1

\begin{tabular}{|c|c|c|c|c|c|c|}
\hline \multicolumn{7}{|c|}{ Proportion Correct } \\
\hline \multirow[b]{2}{*}{ Age } & \multicolumn{3}{|c|}{ Take-out Tasks } & \multicolumn{3}{|c|}{ Put-in Tasks } \\
\hline & 6-container & 12-container & Mean & 6-container & 12-container & Mean \\
\hline 2 & $\underline{0.78}$ & $\underline{0.57}$ & 0.68 & 0.53 & 0.37 & 0.45 \\
\hline 3 & $\overline{0.85}$ & $\overline{0.76}$ & 0.81 & $\overline{0.80}$ & $\overline{0.58}$ & 0.68 \\
\hline Mean & $\overline{0.82}$ & $\overline{0.67}$ & & $\overline{0.67}$ & $\overline{0.48}$ & \\
\hline \multicolumn{7}{|c|}{ Adjacency } \\
\hline 2 & 0.74 & 0.74 & 0.74 & 0.55 & 0.38 & 0.48 \\
\hline 3 & 0.90 & 0.81 & $\overline{0.85}$ & 0.82 & 0.63 & $\overline{0.73}$ \\
\hline Mean & $\underline{0.82}$ & $\underline{0.78}$ & & $\underline{0.69}$ & $\underline{0.50}$ & \\
\hline
\end{tabular}

$(\mathrm{MSE})=0.051, P<0.001 ;$ of task $(\mathrm{F}(1,22)=23.57, \mathrm{MSE}=0.087$, $P<0.001$; and of array $(\mathrm{F}(2,44)=11.09, \mathrm{MSE}=0.44, P<0.001$. There were lower proportions correct in 12 - versus 6 -container arrays $(0.57$ vs. 0.74), as expected given an hypothesised greater difficulty of keeping track of more versus fewer items. As hypothesised, performance was better on the linear array $(0.74)$ than on either the circular $(0.63)$ or random $(0.60)$ arrays (Fisher's Least Significant Difference Test, $P<0.05$, here and in the following). The lack of a difference between circular and random arrays suggests that children were able to treat random arrays of such small numbers of instances as reasonably orderly-essentially like a circle. That take-out tasks were easier $(0.74)$ than put-in $(0.57)$ was consistent with our intent to disrupt adjacent neighbour response tendencies in the latter. There was also a significant age $\times$ task $\times$ number interaction $-F(1,22)=$ $5.94, \mathrm{MSE}=0.026, P<0.025$. The means for that interaction are those underlined in the top of Table 1 . In essence, only on the easiest problems did two- and three-year-olds perform equally, while two-year-olds were more influenced than three-year-olds by effects of task and number. Thus, two-year-olds were worse than three-year-olds except on take-out, sixitem problems; put-in tasks were more difficult than take-out, except for three-year-olds on six-item problems; and twelve containers were more difficult than six, except for three-year-olds on take-out problems.

The effects of age in this analysis are unremarkable; they are as expected given that two-year-olds were not trying deliberately to consider each instance whereas three-year-olds were. However, the effects for task, array, and number remain if three-year-olds' scores are analysed separately. 
Proportion-correct is a measure of success or outcome. How did children achieve this outcome? That is, what strategies were they using to keep track of instances considered? First, there was no evidence that children were using a memory-only strategy since, following Potter and Levy's reasoning, performance on the random arrays was not better than that on the systematic arrays at either age. However, children's use of a memoryonly strategy is better revealed by their errors. Remembering those containers previously considered should reduce redundancies but, since this does not protect against omitting a container altogether, it should yield relatively more exclusions. Both sorts of errors occurred in equal proportions for all problem types and ages; the proportion of the total containers never considered (exclusions) was 0.17 , and the proportion of total acts considering the same container again (redundancies) was 0.18 .

Whether children were employing a sequencing strategy would be revealed by the pattern of their movements from container to adjacent container in order. Adjacency was defined as the proportion of the total number of acts which constituted transitions from one location to an adjacent location. Adjacency is difficult to logically define for random arrays. ${ }^{3}$ But adjacency is easily and comparably defined for the linear and circular arrays. A 2 (age) $\times 2$ (task) $\times 2$ (array) $\times 2$ (number) ANOVA on adjacency scores yielded effects similar to those in the analysis of proportion correct. In general, factors which influenced success similarly influenced adjacency. Thus, there were effects of age $F(1,21)=9.66$, $\mathrm{MSE}=0.164, \quad P<0.05 ; \quad$ of task $\mathrm{F}(1,21)=54.77, \quad \mathrm{MSE}=0.031$, $P<0.001$; and number $\mathrm{F}(1,21)=15.11, \mathrm{MSE}=0.055, P<0.001$. There was also an age $\times$ task $\mathrm{F}(1,21)=6.29, \mathrm{MSE}=0.31, P<0.02$; task $\times$ number $\mathrm{F}(1,21)=7.20$, MSE $=0.031, P<0.02$; and an uninterpretable four-way interaction. The means for the age $\times$ task and for the task $\times$ number interactions are those underlined in Table 1 . The general similarity in results across proportion-correct and adjacency scores can be seen in that table.

A more precise examination of the relationship between success and adjacency involves correlating the two measures; if sequencing enables one to consider every instance, then adjacency should be correlated with proportion correct. For the take-out task, correlations of proportion correct with adjacency were 0.56 and $0.58(P \mathrm{~s}<0.05, d f=10)$ for the twoand three-year-olds, respectively; for the put-in task they were 0.62 and $0.75(P \mathrm{~s}<0.05, d f=10)$, respectively.

\footnotetext{
${ }^{3}$ This is because in a random array many other locations may be equally close to and, hence adjacent in some sense, to a single other location.
} 


\section{Discussion}

We defer discussion of the complete results until after Experiment 2. At this point, consider the task differences alone. Children performed quite differently on the put-in and take-out tasks: two-year-olds were unsuccessful at even transparent versions of the put-in task; three-year-old's success was decidedly lower on put-in than take-out tasks. Our intent in using these tasks was that they differ in the extent to which they disrupt considering the instances in sequence. However, it became evident in conducting Experiment 1 that the tasks differed in another fashion as well. Specifically, there were differences in the practical consequences to the child of avoiding errors, especially redundancies. In the take-out task, if the child attempts to empty a previously emptied jar no further pea comes out. The task itself thus contains practical feedback concerning the futility of redundancy. In the put-in task, however, the child could put many pennies into each bank. In spite of instructions to put a penny in every bank, putting all the pennies in some bank might be more like the child's everyday experience with banks. Either or both of these factorsdifferences in error feedback or disruption of sequencing-might account for poorer performance on the put-in as opposed to take-out tasks. If the effect was due to the presence or absence of error feedback rather than the disruption of sequencing, this would undermine our intended interpretation of the tasks.

\section{EXPERIMENT 2}

This second experiment constituted a replication and extension of the first. Four tasks were constructed representing the possible combinations of presence and absence of error feedback crossed with disruption or not of sequencing. In addition, these tasks were all variations of put-in tasks. Of the two tasks used in Experiment 1, the take-out task seemed most similar to counting or search tasks, specifically to search tasks. Any such similarity to searching, or any differential similarity to counting or searching across tasks, was thus eliminated in Experiment 2.

\section{Method}

Subjects. Twelve different three-year-olds (aged 3.0 to 3.6 years, $M=3.2$ years) from the same school were tested.

Tasks. The four tasks all required that the child insert a small stick through a hole into each of 12 , lidded, opaque, film containers. For all tasks the 12 containers were arranged in a circle. This array type and 
number were chosen because in Experiment 1, three-year-olds' performance on these problems was not subject to ceiling effects.

For tasks involving feedback, the hole in each lid opened into a thin vertical tube within the interior of the film canister. Only one stick would fit in each container; attempting to insert a second would cause it to protrude from the top. For tasks without feedback, there was no interior tube and multiple sticks could be easily inserted. For tasks providing disruption of sequencing, the containers were glued to the board. The child him- or herself put the stick into the container, and then reached to the adult for the next stick. Thus for this version, the child's hand was away from the array altogether when it was time to select the next container. For tasks providing no disruption, containers were set in hollow wells on a board. The child had to pick up a container, hold it out to the adult (who would insert a stick), and then replace it in its well. Thus, for this version, at the point when the child selected a new container, his or her hand was at the previous container.

Procedures. As in Experiment 1, each child was first given a series of warm-up trials. Then the child was given one trial on each of the four tasks. Four different orders of presentation were used so that across children, each of the four tasks was administered equally in first through fourth temporal orders. Instructions at the start of each of the tasks alerted the child to who would be inserting the sticks, e.g. "this time you hand the can to me and I'll put the stick in".

\section{Results}

Proportion-correct and adjacency scores were computed, as in Experiment 1. A 2(feedback) $\times 2$ (disruption) ANOVA was conducted on each of these scores. There were effects of disruption on proportion-correct $[\mathrm{F}(1,11)=6.59, \mathrm{MSE}=0.023, P<0.005]$ and on adjacency $[\mathrm{F}(1,11)=$ 4.73, MSE $=0.029, P<0.05]$. Effects due to feedback were notably absent $(F s<1)$. As indicated in the rationale for this experiment, any potential effects of feedback might manifest themselves most clearly in redundancy errors. However, a 2 (feedback) $\times 2$ (disruption) ANOVA on redundancy errors also revealed only an effect of disruption $[\mathrm{F}(1,11)=14.30, \mathrm{MSE}=0.044, P<0.005]$ not feedback $[\mathrm{F}<1]$. Table 2 shows the means for each of the measures. It also shows the comparable means from Experiment 1 which were clearly replicated here.

The rest of the data further corroborate the results from Experiment 1. Proportion-correct correlated with adjacency, $r(10)=0.69, P<0.05$. And, an analysis of children's self-corrections also confirmed Experiment 
TABLE 2

Comparisons of the Different Tasks in Experiments 1 and 2 on the Different Measures ${ }^{1}$

\begin{tabular}{|c|c|c|c|c|}
\hline $\begin{array}{l}\text { Experiment } 2 \\
\text { Experiment } 1\end{array}$ & $\begin{array}{c}\text { No-disruption; } \\
\text { Feedback } \\
\text { Take-out }\end{array}$ & $\begin{array}{l}\text { No-disruption; } \\
\text { No-feedback }\end{array}$ & $\begin{array}{l}\text { Disruption; } \\
\text { Feedback }\end{array}$ & $\begin{array}{c}\text { Disruption; } \\
\text { No-feedback } \\
\text { Put-in }\end{array}$ \\
\hline \multicolumn{5}{|c|}{ Proportion Correct: } \\
\hline Experiment 2 & $\underline{0.62}$ & 0.56 & 0.47 & $0.44^{2}$ \\
\hline Experiment 1 & 0.76 & & & $0.54^{2}$ \\
\hline \multicolumn{5}{|l|}{ Adjacency: } \\
\hline Experiment 2 & $\underline{0.80}$ & 0.76 & 0.72 & $0.56^{2}$ \\
\hline Experiment 1 & 0.77 & & & $0.63^{2}$ \\
\hline \multicolumn{5}{|l|}{ Redundancy: } \\
\hline Experiment 2 & $\underline{0.12}$ & 0.18 & 0.38 & $0.37^{2}$ \\
\hline Experiment 1 & $\underline{0.12}$ & & & $0.25^{2}$ \\
\hline
\end{tabular}

\footnotetext{
${ }^{1}$ Scores for Experiment 1 are reported only for three-year-olds' performance on the 12-item, circular, unmarked array in order to be comparable with Experiment 2.

${ }^{2}$ Means appearing in one row adjoined by a line are not significantly different, as indicated by the relevant overall ANOVA; if not joined, they are different. Thus, for example, in Experiment 2 there were significant main effects of disruption but no effects of feedback on all three measures.
}

$1 ; 88 \%$ of all self-corrections avoided a redundancy, which is significantly greater than expected by chance $(P<0.05)$.

\section{Discussion}

Differences between the tasks in disruption of sequencing accounted for differences in task performance. This confirms and extends the intended interpretation of the task differences in Experiment 1. The tasks used for this experiment also avoid any possible problems in Experiment 1 caused by some similarity of the take-out task to search tasks. They further avoid any problems with children's looking that may have occurred in Experiment 1 (see Footnote 1).

\section{GENERAL DISCUSSION}

The findings on opaque problems parallel those of Gelman and Gallistel (1978), Wellman et al. (1984), and Potter and Levy (1968). Three-yearolds were reasonably successful at considering every instance for small problem sizes and for regular (in this case linear) arrays. Their performance systematically deteriorated with irregular and/or larger arrays. More importantly, the data suggest that three-year-olds' success resulted from deliberately attempting to consider every instance once, as instructed, 
and employing a sequencing strategy to achieve this goal. Two-year-olds' more limited success seems accounted for by an incidental tendency to move along such arrays from one location to its adjacent neighbours.

To elaborate briefly, consider first the data on whether children attempted to consider every instance at all. Even on transparent problems two-year-olds achieved success only on take-out problems, where sequencing tendencies were not obstructed. Three-year-olds, however, were consistently successful on both sorts of transparent problems. On opaque problems they evidenced deliberate self-corrections and high (albeit not equal) levels of adjacency on put-in as well as take-out problems. These patterns of performance were replicated for three-year-olds in Experiment 2 . The results, thus, show that quite young children, three-year-olds, easily understand simple instructions to consider every instance even for a variety of relatively arbitrary tasks. The data further show that they meet such demands by utilising a quite reasonable and deliberately employed strategy, namely, imposing a sequential order on one's movements so that items are considered in order. The relevant findings here are the predicted disruption (of adjacency and success) caused by several task manipulations, coupled with significant correlations of adjacency with success. Such young children were not always able to flawlessly execute a sequencing strategy, hence actually considering every available instance varied predictably in relation to several factors, such as the number of items to be sequenced and the spatial configurations of the arrays.

In total, we find that quite young children: (1) can deliberately consider every available instance; (2) can do so in tasks unlike counting and searching. What is the significance of these two demonstrations? That young children employ sequencing strategies in order to systematically consider every available instance was previously suspect; in most earlier research (Gelman \& Gallistel, 1978; Potter \& Levy, 1968; Wellman et al., 1984) success may have resulted from a simple adjacent neighbour response tendency (but see Gelman \& Meck, 1983). The validity of this alternative explanation is underscored since it appears to account for two-year-olds' performance on the present tasks. It is of course possible that two-year-olds may be more knowledgeable about the goal of and strategies for considering every instance than our studies indicate. Nevertheless the positive results for young three-year-olds $(M=3.3)$ remain. These results, like recent research on young children's employment of memory and search strategies (DeLoache, 1984; Wellman \& Somerville, 1982) contribute to an emerging picture of the deliberate, strategic problem solving skills of young preschoolers and toddlers.

The specific nature of the problem-solving skill studied here is also of importance. Considering every available instance can be analysed as a simple form of an important family of problem solving skills- 
consideration of multiple alternatives - that has been previously studied only in its later varieties with older children (e.g. Inhelder \& Piaget, 1958; Seigler \& Liebert, 1975). It is usually claimed that the value of these later skills is that they allow the reasoner to proceed systematically instead of capriciously and haphazardly. Our claim is that simpler varieties within this family partake of the same general advantage. In many reasoning and problem-solving situations one needs to proceed, in order, exhaustively, progressively, through a series of facts, instances, possibilities, or choices. Skill at considering every available instance does not guarantee all this systematicity; it is, however, a plausible first step. Hence the importance of ascertaining when it is acquired. The earlier the age at which systematic consideration of alternatives is available in some form, then the more likely it is to prove a potent contributor to as well as product of cognitive development.

Implicit in the above is an attribution to young children of general problem-solving skills and reasoning abilities. Such a description is at odds, at least in emphasis, with an increasingly prevalent description of the young child as a collection of quite limited problem solving devices. For example, in the latest Handbook of Child Psychology, Brown, Bransford, Ferrara and Campione (1983) suggest that early skills are welded to constrained domains and are context-bound; Gelman and Baillargeon (1983) suggest that early knowledge is represented in a form that prevents it from being accessed, utilised, and worked on more generally. These notions allow one to account for the rich, but the uneven character of young children's competencies, by viewing early skills as the acquisition of task-specific expertise; e.g. skill at counting or searching per se rather than at solving problems or reasoning logically more generally.

A claim that component $A$ is embedded in larger skill $B$ would, at the least, seem to mean that $A$ is executed only if $B$ is. If we take $B$ to be either searching or counting and $A$ to be considering every instance, then the present data suggest that by three years of age considering every instance is at least partly free of such embeddedness. Skill at considering every available instance is not purely a captive subroutine of counting or searching, but apparently recruited quite easily for other tasks, even the relatively arbitrary tasks utilised in the present research. Consider in this regard especially the tasks of Experiment 2, which were constructed for reasons of experimental design and not because of their familiarity to young children. One might argue that putting in and taking out are also naturally-occurring skill accomplishments of young children. However, everyday varieties of these tasks do not require putting in or taking out one and only one item, akin to counting effectively or searching efficiently. It is this specific aspect of the child's performance that concerns us in the present work, not simply putting or taking items into and out of repositories. 
The general issue here is a difficult one. One problem with attempts to find general skills in young children is that they must manifest themselves in particular tasks. For young children to perform on the tasks at all, these must be familiar to them. Thus, one can always argue that a putative general skill is actually a cluster of specific ones, learned by repeated practice in specific familiar situations. There is no simple methodological fix for this concern. Nevertheless, one can attempt to construct reasonably familiar tasks which, in the crucial respect, are arbitrary. We have attempted to do this by using take-out and put-in tasks of several different varieties, but by asking the child to take-out and put-in items once and only once. This is a requirement which is not typically a part of emptying and filling endeavours, though it is typically a part of counting and perhaps of searching.

It might also be argued that since children were instructed to consider every instance once, they merely did as they were told. However, that is the point: simple instructions sufficed for young three-year-olds to adopt this goal and to recruit in these situations an effective sequencing strategy. Such a goal (doing each once) may be familiar to them because of its status as a subgoal in counting and searching, but nonetheless young children were easily able to adopt it more generally on our tasks and to recruit an appropriate strategy for its fulfilment.

Our claim is not that the view of young children as a collection of specific purpose devices is completely or even largely wrong, only that the complementary processes that result in more generalised access and more general purpose skills are also in place quite early. At some point limitedaccess, task-specific skills become inefficient as more is learned and similar facts or subroutines must be represented and stored many times within many larger routines. We claim this point is reached earlier rather than later in development. Relatedly, we suggest that current research on counting and searching in young children informs us not only about the development of counting and searching, but about the origins of reasoning and problem solving skills more generally.

Manuscript received 6 October 1986.

\section{REFERENCES}

Antell, S. E. \& Keating, D. P. (1983). Perception of numerical invariance in neonates. Child Development, 54, 695-701.

Beckwith, M. \& Restle, F. (1966). Process of enumeration. Psychological Review, 73, 437-444.

Brown, A. L., Bransford, J. D., Ferrara, R. A., \& Campione, J. C. (1983). Learning, remembering, and understanding. In J. H. Flavell \& E. M. Markman (Eds), Handbook of child psychology, volume III: Cognitive development. New York: John Wiley. 
Cornell, E. H. \& Heth, C. D. (1983). Spatial cognition: Gathering strategies used by preschool children. Journal of Experimental Child Psychology, 35, 93-110.

DeLoache, J. S. (1984). Oh where, oh where: Memory-based searching by very young children. In C. Sopian (Ed.), Origins of cognitive skills. Hillsdale, N.J.: Lawrence Erlbaum Associates Inc.

Gelman, R. \& Baillargeon, R. (1983). A review of some Piagetian concepts. In J. H. Flavell \& E. M. Markman (Eds), Handbook of child psychology, volume III: Cognitive development. New York: John Wiley.

Gelman, R. \& Gallistel, C. R. (1978). The child's understanding of number. Cambridge, Mass.: Harvard University Press.

Gelman, R. \& Meck, E. (1983). Preschoolers' counting: Principles before skill. Cognition, 13, 343-359.

Inhelder, B. \& Piaget, J. (1958). The growth of logical thinking: From childhood to adolescence. London: Routledge \& Kegan Paul.

Potter, M. C. \& Levy, E. I. (1968). Spatial enumeration without counting. Child Development, 39, 265-273.

Seigler, R. S. \& Liebert, R. M. (1975). Acquisition of formal scientific reasoning by $10-$ and 13-year-olds: Designing a factorial experiment. Developmental Psychology, 11, 401-402.

Starkey, P., Spelke, E., \& Gelman, R. (1980). Number competence in infants. Paper presented at the International Conference on Infant Studies, New Haven, CT.

Wellman, H. M. \& Somerville, S. C. (1982). The development of human search ability. In M. E. Lamb \& A. L. Brown (Eds), Advances in developmental psychology, Vol. II. Hillsdale, N.J.: Lawrence Erlbaum Associates Inc.

Wellman, H. M., Somerville, S. C., Revelle, G. L., Haake, R. J., \& Sophian, C. (1984). The development of comprehensive search skills. Child Development, 55, 472-481. 